\section{Zylinder im Urin}

W. G. Guder

München, Deutschland

Synonym(e) Harnzylinder

Englischer Begriff urine casts

Definition Bildung von zylindrischen Ausscheidungsprodukten, die im $>$ Harnsediment als typische Bestandteile der renalen Mukoproteinausscheidung imponieren.

Struktur Harnzylinder entstehen durch Ausscheidung von - Tamm-Horsfall-Protein aus den luminalen Membranen des dicken aufsteigenden Teils der Henle-Schleife mit oder ohne Einlagerung (Hyaline Zylinder) zellulärer oder mikrobiologischer Komponenten.

Pathophysiologie Während hyaline Zylinder durch verstärkten distalen Harnfluss auch unter physiologischen Bedingungen im Harn vorkommen, bedeutet jede pathologische Einlagerung von Epithelzellen, > Erythrozyten oder Leukozyten ( $\triangleright$ Leukozyt) sowie $\triangleright$ Bakterien einen beweisenden Hinweis auf pathologische Vorgänge im tubulären Apparat der Niere.

Untersuchungsmaterial Erster Morgenurin.

Analytik Mikroskopische Analyse bei niedriger $(\times 100)$ oder starker $(\times 400)$ Vergrößerung vorzugsweise im Phasenkontrast. Neuerdings ist auch eine mechanisierte Quantifizierung von Zylindern im Harn mit einer Durchflussdigitalpho- tographischen Methode möglich (iQ 200, Iris, UN containing UF 5000/4000, Sysmex, FUS-2000, Diriu Industrial Co).

Referenzbereich Keine bis einzelne hyaline Zylinder.

Bewertung Pathologisch sind folgende Zylinderarten $\mathrm{zu}$ bewerten:

- Epithelzylinder

- Leukozytenzylinder

- Erythrozytenzylinder

- Granuläre Zylinder

- Wachszylinder

- Pigmentierte Zylinder

- Bakterienzylinder

- Alle Mischungen derselben

\section{Literatur}

Benovska M, Wiewiorka O, Pinkanova J (2018) Evaluation of FUS-2000 urine analyzer: analytical properties and particle recognition. Scand J Clin Lab Invest. https://doi.org/10.1080/00365513.2017.1423108

DeLange JR, Kouri T, Huber AR, Hannemann-Pohl K, Guder WG, Lun A, Sinha P, Stamminger G, Baier L (2000) The role of automated urine particle flow cytometry in clinical practice. Clin Chim Acta 301:1-18

Fogazzi GB, Ponticelli C, Ritz E (1999) The urinary sediment, an integrated view, 2. Aufl. Oxford University Press, Oxford

Kouri T, Fogazzi G, Gant V, Hallander H, Hofmann W, Guder WG (2000) European urinalysis guidelines. Scand J Clin Lab Invest 60(Suppl):231

Shayanfar N, Tobler U, von Eckardstein A, Bestmann L (2007) Automated urinalysis: first experiences and a comparison between the Iris iQ200 urine microscopy system, the Sysmex UF-100 flow cytometer and manual microscopic particle counting. Clin Chem Lab Med $45: 1251-1256$ 